\title{
0 uso de plantas medicinais para doenças parasitárias
}

\author{
Cinthia Rodrigues Melo ${ }^{a^{*}}$, Andressa Brito Lira ${ }^{a b}$, Mateus Feitosa Alves ${ }^{c}$, Caliandra Maria Bezerra \\ Luna Lima ${ }^{\text {ade }}$ \\ ${ }^{a}$ Laboratório de Ensaios Toxicológicos do Instituto de Pesquisa em Fármacos e Medicamentos, Universidade Federal da Paraíba, \\ João Pessoa, 58051-970, Brasil. *cinthiamelo_rm@hotmail.com \\ b Programa de Pós-Graduação em Produtos Naturais e Bioativos Sintéticos, Universidade Federal da Paraíba, João Pessoa, 58051- \\ 970, Brasil. \\ c Departamento de Ciências Farmacêuticas do Centro de Ciências em Saúde, Universidade Federal da Paraíba, 58051-970, Brasil. \\ ${ }^{\mathrm{d}}$ Departamento de Fisiologia e Patologia do Centro de Ciências em Saúde, Universidade Federal da Paraíba, João Pessoa, 58051-970, \\ Brasil. \\ ${ }^{\text {e }}$ Programa de Pós-Graduação Interdisciplinar em Modelo de Decisão e Saúde. Universidade Federal da Paraíba, João Pessoa, 58051 - \\ 970, Brasil.
}

Recebido: 12 dezembro 2016 / Aceito: 4 janeiro 2017 / Publicado online: 15 fevereiro 2017

\begin{abstract}
Resumo
O uso de plantas para tratamento de muitas enfermidades vem do conhecimento de antepassados e se propaga ao longo dos anos, mostrando ser uma boa alternativa, principalmente para aqueles que possuem poucos recursos financeiros. Dentre as doenças tratadas encontram-se as parasitoses que são a causa de mais de 200 mil mortes por ano. Esta revisão sistemática pesquisou, na literatura, quais espécies vegetais são usadas por diferentes comunidades do mundo para o tratamento de parasitoses. Para isso foram utilizados os bancos de dados primários: PubMed, SciELO e LILACS. Os termos usados na busca encontraram-se na língua inglesa, associados com o operador booleano AND: "parasitic diseases" AND "plants medicinal". Encontrando-se 853 publicações, sendo posteriormente aplicados critérios de inclusão e exclusão, selecionando ao todo 9 artigos. Foram verificadas 67 espécies de 42 famílias, na qual a família Leguminosae foi a mais citada pela população, e as espécies vegetais mais usadas foram Azadirachta indicam, Euphorbia abyssinica e Mangifera indica, com destaque para a primeira. A malária foi a que teve maior número de plantas medicinais para o seu tratamento. Apesar do desenvolvimento de fármacos, observa-se que a medicina tradicional não caiu em desuso, buscando incentivar estudos científicos que garantam a eficácia terapêutica destas plantas medicinais.
\end{abstract}

Palavras-chave: Azadirachta indicam, etnobotânica, plantas, parasitoses.

\section{The use of medicinal plants for parasitic diseases}

\begin{abstract}
The use of plants for the treatment of many diseases comes from the knowledge of ancestors and propagates over the years, proving to be a good alternative, especially for those with low financial resources. Among the diseases treated are the parasitic diseases that are the cause of more than 200 thousand deaths per year. This systematic review search for in the literature plant species used by different communities of the world for the treatment of parasitoses. Were used primary databases: PubMed, SciELO e LILACS. The terms used in the search were found in the English language, associated with the Boolean operator AND: "parasitic diseases" AND "plants medicinal". Finding 853 publications, after which inclusion and exclusion criteria were applied, selecting of 9 articles. Were found 67 species of 42 families, in which the Leguminosae family was the most cited by the population, and the most used plant species were Azadirachta indica, Euphorbia abyssinica and Mangifera indica, with featured on the first. Malaria had a more number of medicinal plants for its treatment. Even with drug development, it can be observed that traditional medicine hasn`t fallen into disuse, encouraging scientific studies that ensure the therapeutic efficacy of these medicinal plants.
\end{abstract}

Keywords: Azadirachta indicam, ethnobotany, plants, parasitoses.

\section{Introdução}

As plantas são fontes naturais, compostas por uma variedade de metabólitos que lhes concedem diversas propriedades farmacológicas. E é justamente por apresentar diferentes efeitos terapêuticos, que as plantas são usadas pela população como fonte de tratamento de diferentes doenças (Morais et al., 2016).

$\mathrm{O}$ consumo de plantas medicinais tem base no 
conhecimento tradicional, que é repassado ao longo dos anos pelas pessoas mais idosas, tornando-se comum o uso desse meio para o tratamento de sintomas de algumas doenças. Sendo também, uma alternativa para pessoas com menores recursos financeiros, já que o tratamento por espécies vegetais tem menor custo e é mais acessível. Sabe-se que existem inúmeras espécies vegetais espalhadas pelo mundo, muitas destas ainda sem estudos científicos que comprovem sua eficácia terapêutica. Portanto é de interesse científico esse conhecimento popular, pois através deste, se tem uma fundamentação para a busca investigativa das propriedades farmacológicas das diferentes espécies, e assim poder registrá-las formalmente (Balbinot et al., 2013; Messias et al., 2015).

Existem estudos que comprovam a eficácia terapêutica que muitas plantas medicinais possuem, sendo muitas vezes usadas não só para tratamento, como também para a prevenção de doenças. Apesar de apresentarem inúmeros benefícios, é necessário que se tenham estudos toxicológicos para garantir o uso dessas fontes naturais, já que estas são compostas por inúmeras substâncias podendo algumas delas ter efeito maléfico para saúde (Bochneret al., 2012; Costa et al., 2012; Guedes et al., 2012).

Dentre as doenças tratadas por plantas medicinais, encontra-se às causadas por parasitas. Estudos mostram que cerca de 300 espécies de helmintos, e mais de 70 espécies de protozoários já foram relatados em humanos (Edrissian et al., 2016). Sendo, portanto as infecções parasitárias a causa da morte de aproximadamente 200 mil pessoas por ano (Bahmani et al., 2014), principalmente quando estas não são tratadas de forma adequada (Sousa et al., 2013).

A malária é uma das principais doenças parasitárias, afetando mais de dois bilhões de pessoas no mundo (Klein, 2013). Bem como as parasitoses intestinais: ascaridíase, tricuríase, ancilostomíase, amebíase e giardíase, com alta prevalência mundial (Visser et al., 2011). Estas enfermidades são comuns, principalmente naqueles países subdesenvolvidos, e o uso de plantas medicinais para o tratamento das parasitoses é uma ação corriqueira entre a população que tem baixa renda, ou o acesso ao medicamento é inviável. Assim, o conhecimento advindo de seus ancestrais, se torna uma ferramenta para sanar os problemas de saúde (Oliveira et al., 2010).

Portanto, é importante a investigação de quais espécies vegetais são usadas popularmente para o tratamento de enfermidades como às parasitoses. Visto que estas acometem um grande número de pessoas, e principalmente aquelas de menor poder econômico, sendo essa terapêutica uma alternativa viável para tais indivíduos.

\section{Material e Métodos}

Para o levantamento bibliográfico das plantas medicinais utilizadas pela população, para o tratamento de doenças parasitárias, foram utilizados os bancos de indexadores de base de dados primários: PubMed, SciELO e LILACS. Os termos utilizados na busca com o operador booleano AND foram: "parasitic diseases" AND "plants medicinal".

Os critérios de inclusão das pesquisas foram: publicação nos últimos cinco anos (2012-2016); livre acesso (open source) e que relacionavam doenças parasitárias e plantas medicinais. Os critérios de exclusão foram: versões duplicadas de publicações em uma mesma base de dados ou entre bases diferentes; artigos de revisão; estudos que não citam a planta usada para o tratamento da doença parasitária e publicações que tratavam de estudos sobre plantas medicinais usadas para o tratamento de doenças parasitárias em animais.

\section{Resultados e Discussão}

Apesar do avanço científico, a medicina tradicional ainda tem sido um tratamento bastante utilizada. $\mathrm{O}$ uso de plantas medicinais para o controle de mosquitos vetores de doenças parasitárias era uma atitude antiga das comunidades (Innocent et al., 2014). Como exemplo da influência da cultura indígena, que se perpetua de geração em geração (Lulekal et al., 2013).

O tratamento por meio de plantas medicinais é considerado, por muitas pessoas, mais seguro que drogas sintéticas, justamente por ser um produto natural. Estudos revelam que cerca de $8 \%$ de todas as admissões hospitalares nos Estados Unidos são devido a reações adversas a drogas sintéticas, pelo menos 100.000 pessoas/ano morrem por intoxicações medicamentosas (Nasri e Shirzad, 2013). Sabendo da importância e dos benefícios trazidos pelo uso de produtos naturais, país como a China tem incorporado no componente formal de sistema de saúde, sistemas tradicionais no tratamento de doenças (Limenih et al., 2015).

Inicialmente foram identificados 853 estudos, após aplicação dos critérios de inclusão foram selecionados 35 (trinta e cinco) artigos indexados no PubMed, 4 (quatro) artigos no SciELO e 4 (quatro) no LILACS. Em seguida houve a aplicação dos critérios de exclusão, sendo escolhidos 9 (nove) artigos. Os dados desses artigos, das espécies de plantas utilizadas pela população para tratamento de doenças parasitárias, estão descritos na Tabela 1.

Foram identificadas 67 espécies usadas para o tratamento de parasitoses, distribuídas em 43 famílias. Dentre as plantas citadas pela população, a família Leguminosae foi a mais freqüente 16,3\%, seguida das famíias Meliaceae $(11,6 \%)$, Solanacea (11,6\%), Compositae (9,3\%) e Euphorbiaceae $(9,3 \%)$. Dentre as doenças parasitárias, a malária foi a que teve maior número de plantas medicinais para o seu tratamento. As espécies de plantas mais citadas foram: A. indicam, $E$. abyssinica e $M$. indica.

Em alguns artigos, não foi discriminado o tipo de parasita que determinada espécie era usada (Innocent et al., 2014). A malária acomete um grande número de pessoas no mundo, sobretudo nas nações em desenvolvimento e subdesenvolvidas (Gomes et al., 2011; Andrews et al., 2014). Por isso muitos estudos têm sido realizados sobre o uso de plantas medicinais que venham tratar esta doença (Singh e Singh, 2014).

Os locais em que foram realizadas as pesquisas eram comunidades do interior dos países e apresentavam condições socioeconômicas baixas, e com pouco acesso aos serviços de saúde, o que refletia na forma de tratamento das doenças parasitárias pelos moradores de cada região. Encontrando na medicina tradicional a alternativa de tratamento mais acessível do que por medicamentos industrializados (Tabuti et al., 2012). 
Tabela 1. Plantas medicinais utilizadas no tratamento de parasitoses.

\begin{tabular}{|c|c|c|c|c|c|}
\hline Planta Utilizada & Família & Uso & Planta Utilizada & Família & Uso \\
\hline \multicolumn{3}{|c|}{ Abera (2014), Etiópia } & \multicolumn{3}{|c|}{ Malan et al. (2015), Costa do Marfim } \\
\hline Allium sativum & Liliaceae & Malária & Alchornea cordifolia & Euphorbiaceae & Malária \\
\hline Opuntia fícus-indica & Cactaceae & Malária & Alstoni aboonei & Apocynaceae & Malária \\
\hline Croton macrostachyus Del & Euphorbiaceae & Malária & Anthocleista nobilis & Gentianaceae & Malária \\
\hline Euphorbia abbyssinica JF* & Euphorbiaceae & Ascaridíase & Azadirachta indica* & Meliaceae & Malária \\
\hline Tavernier aabyssinica A.Rich & Leguminosae & Parasitas internos & Bambusa vulgaris & Poaceae & Malária \\
\hline Glinus lotoides & Molluginaceae & Vermes & Bidens pilosa $L$. & Compositae & Malária \\
\hline Maisa lanceolata & Myrsinaceae & Filariose & Blighia sapida Koenig & Sapindaceae & Malária \\
\hline Securidica longipedunculata Fresen & Polygalaceae & Parasitas intestinais & Blighia welwitschii & Sapindaceae & Malária \\
\hline Bruceaanti dysenterica Fresen & Simaroubaceae & Parasitas externos & Bridelia micrantha & Phyllanthaceae & Malária \\
\hline Withania somnifera L Dunal & Solanaceae & Malária & Caesalpinia bonduc (L.) & Leguminosae & Malária \\
\hline Zingiber officinalroscoe & Zingiberaceae & Parasitas & Cajanus cajan (L.) Roxb & Leguminosae & Malária \\
\hline \multicolumn{3}{|c|}{ Belayneh et al. (2012), Etiópia } & Carica papaya $L$. & Caricaceae & Malária \\
\hline Aloepirottae & Asphodelaceae & Malária & Cassia alata $L$. & Leguminosae & Malária \\
\hline Azadirachta indica* & Meliaceae & Malária e vermes intestinais & Cassia occidentalis $L$. & Leguminosae & Malária \\
\hline Tamarindus indica & Fabaceae & Parasitas intestinais Malária & Cola lateritiavar.maclaudi & Malvaceae & Malária \\
\hline Balanite saegyptiaca & Zygophyllaceae & Malária & Eclipta prostrata (L.) L. & Compositae & Malária \\
\hline Acacia robusta & Fabaceae & Malária & Flagellaria guineenses Schumach & Flagellariaceae & Malária \\
\hline Acokanthera schimperi & Apocynaceae & Malária & Halle aledermannii (K. Krause) & Rubiaceae & Malária \\
\hline Euphorbia abbyssinica JF* & Euphorbiaceae & Malária & Harungana madagascariensis Lam. & Hypericaceae & Malária \\
\hline \multicolumn{3}{|c|}{ Briones-Chavez et al. (2013), Peru } & Lophiraalata Banks exGaertn. $f$. & Ochnaceae & Malária \\
\hline Ficus insipida & Moraceae & Helmintos & Mangifera indica L.* & Anacardiaceae & Malária \\
\hline \multicolumn{3}{|c|}{ Innocent (2014), Tanzânia } & Momordica charantia $L$. & Cucurbitaceae & Malária \\
\hline Azadirachta indica* & Meliaceae & Filariose/Malária & Musa paradisiaca L. & Musaceae & Malária \\
\hline Annonas quamosa & Annonaceae & Filariose/Malária & Ocimum gratissimum $L$. & Lamiaceae & Malária \\
\hline Annonas enegalensis & Annonaceae & Filariose/Malária & Pentaclethra macrophylla & Leguminosae & Malária \\
\hline Citrus limonium & Rutaceae & Filariose/Malária & Phyllanthus amarus & Phyllanthaceae & Malária \\
\hline Ocimum suave & Lamiaceae & Filariose/Malária & Pterocarpus santalinoides & Leguminosae & Malária \\
\hline Anacardium occidentale & Anacardiaceae & Filariose/Malária & Rauvolfia vomitoria & Apocynaceae & Malária \\
\hline Mangifera indica & Anacardiaceae & Filariose/Malária & Solanum nigrum $L$. & Solanaceae & Malária \\
\hline Psidum guajava & Myrtaceae & Filariose/Malária & Spathodea campanulata & Bignoniaceae & Malária \\
\hline Cocos nucifera & Arecaceae & Filariose/Malária & Vernonia amygdalina & Compositae & Malária/ Helmintíase \\
\hline Annona muricata & Annonaceae & Filariose/Malária & Vernonia confertaBenth. & Compositae & Malária \\
\hline \multicolumn{3}{|c|}{ Kabir et al. (2014), Bangladesh } & Zanthoxylum gilletii & Rutaceae & Malária \\
\hline Andrographis paniculata Nees & Acanthaceae & Malária & Solanum etiopicum & Solanaceae & Vermes \\
\hline Azadirachta indica* & Meliaceae & Helmintíase & Solanum indicum & Solanaceae & Helmintíase \\
\hline \multicolumn{3}{|c|}{ Lulekal et al. (2013), Etiópia } & Solanum torvum & Solanaceae & Helmintíase \\
\hline Hagenia abyssinica & Rosaceae & Parasitas & Mormodica charantia & Cucurbitales & Helmintíase \\
\hline Thalictrum rhynchocarpum & Ranunculaceae & Parasitas & \multicolumn{3}{|c|}{ Ngarivhume et al. (2014), Zimbábue } \\
\hline Embelias chimperi Vatk & Primulaceae & Parasitas & \multirow{4}{*}{$\begin{array}{l}\text { Cassia abbreviata Oliv } \\
\text { Aristolochia álbida Dusch } \\
\text { Toddalia asiatica }\end{array}$} & Fabaceae & \multirow{4}{*}{$\begin{array}{l}\text { Malária } \\
\text { Malária } \\
\text { Malária }\end{array}$} \\
\hline Jasminum abyssinicum Hachs & Oleaceae & Parasitas & & Aristolochiaceae & \\
\hline $\mathrm{Tak}$ & al. (2012), Ugand & & & Rutaceae & \\
\hline Azadirachta indica* & Meliaceae & Malária & & & \\
\hline
\end{tabular}


Foram usadas as plantas: A. indica, Annona squamosa, Annona senegalesis, Citrus limonium, Ocimum suave, Anacardium occidentale, $M$. indica, Psidium guajava, Cocos nucifera e Annona muricata, para combater mosquitos transmissores da malária e elefantíase, sendo relatadas pela população de quatro comunidades da Tanzânia.

P. guajava, C. nucifera, A. Muricata. Os entrevistados não especificaram quais plantas eram utilizadas para cada doença (malária ou elefantíase), mas afirmaram que as folhas e as raízes eram as partes mais utilizadas (Innocent et al., 2014).

Diferentes doenças parasitárias (algumas não especificadas qual a parasitose) foram citadas pelos moradores de uma comunidade da Etiópia e para cada espécie foi relatada uma enfermidade diferente: Allium sativum (malária); Opuntia fícus-indica (vetores da malária); Crotonma crostachyus (malária); E. abyssinica (áscaris); Taverniera abyssinica (parasita interno); Glinus lotoides (verme); Maisa lanceolata (elefantíase); Securidica longipedunculata (parasita intestinal); Brucea antidysentrica (parasita externo); Withania somnifera (malária); Zingiber officinale (tratamento de parasita; Abera, 2014).

Habitantes de um clã no interior de Bangladesh, a partir de conhecimentos advindos de seus curandeiros, fazem uso das seguintes espécies vegetais: Andrographis paniculata (malária) e A. indica (helmintíase; Kabir et al., 2014). Em 4 comunidades de Zimbábue para doenças parasitárias a população citou apenas plantas com propriedades antimaláricas, sendo as raízes as partes mais usadas $(55,3 \%)$ : Cassia abbreviata, Aristolochia albida e Toddalia asiática (Ngarivhume et al., 2014).

A cultura indígena através do uso de plantas medicinais mostrou ser bastante influente na forma de tratamento das doenças parasitárias. Como em algumas regiões da Etiópia em que são mencionadas tais espécies vegetais: Hagenia abyssinica (doenças gastrointestinal e parasitária); Thalictrum rhynchocarpum (doenças gastrointestinal e parasitária); Embelias chimperi (doença gastrointestinal e parasitária); Jasminum abyssinicum (doença gastrointestinal e parasitária; Lulekal et al, 2013).

Em um estudo comparativo entre indígenas e indivíduos colonizados na região do Peru, verificou-se a presença de helmintos intestinais e protozoários. A presença de helmintos na população colonizada foi consideravelmente maior que na população indígena, sendo estes: Ascaris lumbricoides, Ancylostoma duodenalel Necator americanos, Trichuris trichiura, Strongyloides stercoralis. Já os protozoários apresentaram a mesma média em ambas as populações. (Briones-Chávez et al., 2013). Um dos fatores para esse resultado deve-se o uso de plantas medicinais pelos indígenas, plantas estas com possível potencial antihelmíntico como a Ficus insipida, popularmente conhecida como Ojé. Já em comunidades da Uganda foi verificado que, por apresentam poucos recursos financeiros e com pouco acesso aos serviços de saúde, levaram a predominância do uso de conhecimentos adquiridos pelos indígenas (Tabuti et al., 2012).

Uma das consequências da malária é a lesão renal, sendo a causa de $45 \%$ das mortes dos pacientes com a forma grave da doença. Estudos não clínicos mostraram que o extrato bruto da $A$. indica é considerado um candidato potencial para a proteção contra a lesão renal induzida pela malária (Somsak et al., 2015).

A A. indica, possui estudos que a indicam como fonte promissora para desenvolvimento de fármacos antimalárico (Murugan et al., 2016). Estudos apontam atividade favorável em bloquear a ação de vetores como Anopheles coluzzii na transmissão do Plasmodium falciparum (Yerbanga et al,2014). Bem como ação anti-vetorial sobre o Anopheles stephensi, que é considerado um dos principais transmissores da malária (Dembo et al., 2015).

Uma das consequências graves causadas Plasmodium falciparum é a encefalopatia. E achados sugerem que o extrato etanólico de $A$. indica tem efeito protetor sobre o sistema nervoso central inflamado (Bedri et al., 2013).

Entretanto, além da $A$. indica, outras espécies também foram citadas como eficazes para a malária, tais como: Acacia robusta, Aloe Pirottae (malária), Tamarindus indica (parasita intestinal e malária), Balanites aegyptiaca (malária), Acokanthera schimperi e E. abyssinica (malária) (Belayneh et al., 2012).

Em comunidades do litoral leste da Costa do Marfim, 84\% das plantas usadas eram de uso para o tratamento de doenças parasitárias, sendo citadas 33 espécies diferentes (Tabela 1). Entre estas, seis são conhecidas como uso específico contra a malária, são elas: Alstonia boonei, A. indica, Bambusa vulgaris, Harungana madagascariensis, Spathodea campanulata e Vernonia conferta.

A utilidade de alguns fármacos disponíveis está sendo ameaçada pela resistência do parasita à droga (Andrews et al,2014). Portanto, é interessante que seja identificada as plantas que têm sido usadas pela população para o tratamento de doenças, já que estas podem vir a ser potencialmente medicamentos (Lydia et al., 2014).

\section{Conclusões}

O conhecimento repassado ao longo dos anos em cada comunidade sobre o uso de plantas medicinais, para o tratamento de parasitoses, mostrou ainda ser uma realidade. Apesar do desenvolvimento de fármacos eficazes para o tratamento destas doenças (sendo malária a mais citada), a medicina tradicional não ficou em desuso, principalmente, nas comunidades de condições socioeconômicas limitadas.

Foi encontrado que dentre as 67 espécies citadas, $A$. indica, E. abyssinica e $M$. indica foram as que apresentaram maior uso pela população, com destaque para a primeira. A maioria dos estudos foi realizada em países da África, onde doenças como malária e filariose são bastante comuns.

Dessa maneira, os dados reportam a importância da valorização desta forma de tratamento, que se perpetua por muitos anos e apresenta significativa eficácia. Como vem sendo mostrado através de experimentos não clínicos com extrato da $A$. indica, podendo vir a ser um medicamento promissor para o combate a malária.

\section{Referências}

Abera, B. 2014. Medicinal plants used in traditional medicine by Oromo 
people, Ghimbi District, Southwest Ethiopia. Journal of Ethnobiology and Ethnomedicine, 10(40): 1-15.

Andrews K.T.; Fisher G.; Skinner-Adams T.S. 2014. Drug repurposing and human parasitic protozoan diseases. International Journal for Parasitology, 4(2) 95-111.

Bahmani, M.; Saki, K.; Kopaei, M.R.; Karamati, S.A.; Eftekhari, Z.; Jelodari, M. 2014. The most common herbal medicines affecting Sarcomastigophora branches: a review study. Asian Pacific Journ al of Tropical Medicin, 7 (1): S14-S21.

Balbinot, S.; Velasquez, P.G.; Düsman, E.2013. Reconhecimento e uso de plantas medicinais pelos idosos do Município de Marmeleiro - Paraná. Revista. Brasileira de Plantas Medicinais, 15 (4): 632-638.

Bedri, S.; Khalil, E.A.; Khalid, S.A.; Alzohairy, M.A.; Mohieldein, A.; Aldebasi, Y.H.; Etet, P.F.S; Farahna, M. 2013. Azadirachta indica ethanolic extract protects neurons from apoptosis and mitigates brain swelling in experimental cerebral malaria. Malaria Journal, 12: 1-9.

Belayneh, A.; Asfaw, Z.; Demissew, S.; Bussa, N.F. 2012. Medicinal plants potential and use by pastoral and agro-pastoral communities in Erer Valley of Babile Wereda, Eastern Ethiopia. Journal of Ethnobiology and Ethnomedicine, 8 (42): 1-11.

Bochner, R.; Fiszon, J.T.; Assis, M.A.; Avelar, K.E.S. 2012. Problems associated with the use of medicinal plants commercialized in "Mercadão de Madureira", Rio de Janeiro City, Brazil. Revista Brasileira de Plantas Medicinais, 14 (3): 537-547.

Briones-Chávez, C.; Torres-Zevallos, H.; Canales, M.; Stamato, C.M.; O'Riordan, T.G.; Terashima, A. 2013. Differences in prevalence of geohelminth infections between indigenous and settler populations in a remote Amazonian region of Peru. Tropical Medicine \& International Health, 18(5): 615-618

Costa, K.C.S.; Bezerra, S.B.; Norte, C.M.; Nunes, L.M.N.; Olinda, T.M. 2012. Medicinal plants with teratogenic potential: current considerations. Brazilian Journal of Pharmaceutical Sciences, 48 (3): 427-433.

Dembo, E.G.; Abay, S.M.; Dahiya, N.; Ogboi, J.S.; Christophides, G.K.; Lupidi, G.; Chianese, G.; Lucantoni, L.; Habluetzel, A. 2015. Impact of repeated NeemAzal@-treated blood meals on the fitness of Anopheles stephensi mosquitoes. Parasites \& Vector, 8 (94): 1-13.

Edrissian, G.; Rokni, M.B.; Mohebali, M.; Nateghpour, M.; Mowlavi, G.; Bahadori, M. 2016. History of medical parasitology and parasitic infections in Iran. Archives of Iranian Medicine, 19 (8): 601-607.

Gomes, A.P.; Vitorino, R.R.; Costa, A.P.; Mendonça, E.G.; Oliveira M.G.A.; Siqueira-Batista, R. 2011. Malária grave por Plasmodium falciparum. Revista Brasileira de Terapia Intensiva, 23(3): 358-369.

Guedes, A.P.; Franklin, G.; Fernandes-Ferreira, M. 2012. Hypericum sp.: essential oil composition and biologic activities. Phytochemestry Reviews, 11 (1): 127-152.

Innocent, E.; Hassanali, A.; Kisinza, W.N.W.; Mutalemwa, P.P.P.; Magesa, S.; Kayombo, E. 2014. Anti-mosquito plants as an alternative or incremental method for malaria vector control among rural communities of Bagamoyo District, Tanzania. Journal of Ethnobiology and Ethnomedicine, 10(56):1-11.

Kabir, M.H.; Hasan, N.; Rahman, M.; Rahman, A.; Khan, J.A.; Hoque, N.T.; Bhuiyan, R.Q.; Mou, S.M.; Jahan, R.; Rahmatullah, M. 2014. A survey of medicinal plants used by the Deb barma clan of the Tripura tribe of Moulvibazar district, Bangladesh. Journal of Ethnobiology and Ethnomedicine, 10 (19): 1-28.

Klein, E.Y.2013. Antimalarial drug resistance: a review of the biology and strategies to delay emergence and spread. International Journal of Antimicrobial Agents, 41(4): 311-317.

Limenih, Y.; Umer, S.; Wolde-Mariam, M. 2015. Ethnobotanical Study on Traditional Medicinal plants in degadamotworeda, Amhara Region, North Ethiopia. International Journal of Research in Pharmacy and Chemistry, 5(2): 258-273.

Lulekal, E.; Asfaw, Z.; Kelbessa, E.; Damme, P.V. 2013. Ethnomedicinal study of plants used for human ailments in Ankober District, North Shewa Zone, Amhara Region, Ethiopia. Journal of Ethnobiologyand Ethnomedicine, 9 (63):1-13.

Lydia, L.; Lifongo, L.L.; Simoben, C.V.; Ntie-Kang, F.; Babiaka, S.B.; Judson P.N. 2014. A bioactivity versus ethnobotanical survey of medicinal plants from Nigeria, West Africa. Natural Products and Bioprospecting, 4(1):1-19.

Malan, D.F.; Neuba, D.F.R.; Kouakou, K.L. 2015. Medicinal plants and traditional healing practices in ehotile people, around the Aby Lagoon (eastern littoral of Côte d'Ivoire). Journal of Ethnobiology and Ethnomedicine, 11(21): 1-18.

Messias, M.C.T.B.; Menegatto, M.F.; Prado, A.C.C.; Santos, B.R.;
Guimarães, M.F.M. 2015. Popular use of medicinal plants and the socioeconomic profile of the users: a study in the urban area of Ouro Preto, Minas Gerais, Brazil. Revista Brasileira de Plantas Medicinais, 17 (1): 76104.

Morais, N.R.L.; Oliveira Neto, F.B.; Melo, A.R.; Bertini L.M.; Silva, F.F.M.; Alves, L.A. 2016. Prospecção fitoquímica e avaliação do potencial antioxidante de Cnidos colusphyllacanthus (müll. Arg.) Pax\&k.hoffm. Oriundo de apodi - RN. Revista Brasileira de Plantas Medicinais, 18 (1): $180-185$.

Murugan, K.; Panneerselvam, C.; Samidoss, C.M.; Madhiyazhagan, P.; Suresh, U.; Roni, M.; Chandramohan, B.; Subramaniam, J.; Dinesh, D.; Rajaganesh, R.; Paulpandi, M.; Wei, H.; Aziz, A.T.; Alsalhi, M.S.; Devanesan, S.; Nicoletti, M.; Pavela, R.; Canale, A.; Benelli, G. 2016. In vivo and in vitro effectiveness of Azadirachtaindica-synthesized silvernanocrystals against Plasmodium berghei and Plasmodium falciparum, and their potential against malaria mosquitões. Research in Veterinary Science, 106:14-22.

Nasri, H.; Shirzad, H. 2013. Toxicity and safety of medicinal plants. Journal of Herbmed Pharmacology, 2 (2): 21-22

Ngarivhume, T.; Klooster, C.I.E.A.V.; Jong, J.T.V.M.; Westhuizen, J.V. 2014. Medicinal plants used by traditional healers for the treatment of malaria in the Chipinge district in Zimbabwe. Journal of Ethnobiology and Ethnomedicine, 159: 224-237.

Oliveira, G.L.; Oliveira, A.F.M.; Andrade, L.H.C. 2010. Plantas medicinais utilizadas na comunidade urbana de Muribeca, Nordeste do Brasil. Acta Botanica Brasilica, 24(2): 571-577.

Singh, S.; Singh, R. 2014. Herbal medicinal treatment of Malaria in Aliero local government area, Kebbi, Nigeria. Journal of Medicinal Plants Studies, 2(2): 117-126.

Somsak, V.; Chachiyo, S.; Jaihan, U.; Nakinchat, S. 2015. Protective effect of aqueous crude extract of neem (Azadirachta indica) leaves on Plasmodium berghei - induced renal damage in mice. Journal of Tropical Medicine, 15: 1-5.

Somsak, V.; Chachiyo, S.; Jaihan, U.; Nakinchat, S. 2015. Protective effect of aqueous crude extract of neem (Azadirachta indica) leaves on Plasmodium berghei - induced renal damage in mice. Journal of Tropical Medicine, 2015:1-5.

Sousa, R.G.; Falcão, H.S.; Barbosa Filho, J.M.; Diniz, M.F.F.M.; Batista, L.M. 2013. Atividade anti-helmíntica de plantas nativas do continente americano: uma revisão. Revista Brasileira de Plantas Medicinais, 15 (2): 287-292.

Tabuti, J.R.S.; Kukunda, C.B.; Kaweesi, D.; Kasilo, O.M.J. 2012. Herbal medicine use in the districts of Nakapiripirit, Pallisa, Kanungu, and Mukono in Uganda. Journal of Ethnobiology and Ethnomedicine, 8(35): $1-15$.

Visser, S.; Giatti, L.L.; Carvalho, R.A.C.; Guerreiro, J.C.H.2011. Estudo da associação entre fatores socioambientais e prevalência de parasitose intestinal em área periférica da cidade de Manaus (AM, Brasil). Ciência \& Saúde Coletiva, 16(8): 3481-3492.

Yerbanga, R.S.; Lucantoni, L.; Ouédraogo, R.K.; Da, D.F.;Yao, F.A.; Yaméogo, K.B.; Churcher, T.S.; Lupidi, G.; Taglialatela-Scafati, O.; Gouagna, L.C.; Cohuet, A.; Christophides, G.K.; Ouédraogo, J.B.; Habluetzel, A. 2014. Transmission blocking activity of Azadirachta indica and Guierase negalensis extracts on the sporogonic development of Plasmodium falciparum field isolates in Anopheles coluzzii mosquitoes. Parasites \& Vector, 7(185): 1-10. 